\title{
BMJ Open Does sensorimotor function predict graft rupture, contra-lateral injury or failure to return to sports after ACL reconstruction? A protocol for the STOP Graft Rupture study
}

\author{
Anna Cronström (10 , ${ }^{1,2}$ Eva Ageberg, ${ }^{2}$ Charlotte K Häger ${ }^{1}$
}

To cite: Cronström A, Ageberg E, Häger CK. Does sensorimotor function predict graft rupture, contra-lateral injury or failure to return to sports after ACL reconstruction? A protocol for the STOP Graft Rupture study. BMJ Open 2021;11:e042031. doi:10.1136/ bmjopen-2020-042031

- Prepublication history for this paper is available online. To view these files, please visit the journal online (http://dx.doi. org/10.1136/bmjopen-2020042031).

Received 23 June 2020 Revised 26 November 2020 Accepted 14 December 2020

Check for updates

(c) Author(s) (or their employer(s)) 2021. Re-use permitted under CC BY-NC. No commercial re-use. See rights and permissions. Published by BMJ.

${ }^{1}$ Department of Community Medicine and Rehabilitation, Umeå Universitet, Umeå,

Sweden

${ }^{2}$ Department of Health Sciences, Lund University, Lund, Sweden

Correspondence to

Dr Anna Cronström;

anna.cronstrom@umu.se

\section{ABSTRACT}

Introduction People with anterior cruciate ligament (ACL) reconstruction (ACLR) are at high risk of sustaining a graft rupture and/or contra-lateral ACL injury. The main factors that may predispose individuals for subsequent ACL injuries are, however, not established. To reduce the risk of reinjuries, it is of particular interest to identify modifiable risk-factors, for instance, those related to sensorimotor control which are responsive to training. The aim of the current study protocol is to present the design of our prospective cohort study STOP Graft Rupture investigating sensorimotor function as predictors for graft rupture, contra-lateral ACL injury and/or failure to return to sport (RTS) within 3 years following ACLR.

Methods and analysis We aim to recruit 200 individuals (15-35 years, $~ 50 \%$ women) with ACLR from Norrland University Hospital, Umeå and Lund University Hospital, Lund, Sweden. Participants will be assessed with a comprehensive test battery for sensorimotor muscle function, including hop performance, muscle strength, muscle activation, hip and ankle range of motion and postural orientation as well as patient-reported function 1 year after ACLR (baseline). For a subgroup of individuals (Umeå cohort), 3D kinematics and joint position sense will also be evaluated. At follow-up ( $\geq 3$ years post-ACLR), the participants will be asked to answer questions related to new ACL injuries to either knee and about RTS. Separate logistic regression models, adjusting for possible confounders, will be used to evaluate the influence of the different sensorimotor predictors on the prospective outcomes (graft rupture, contra-lateral ACL injury, RTS). Ethics and dissemination This study was approved by the Swedish Ethical Review Board (Dnr 2016/319 and Dnr 2019-04037). The results will be published in international peer-reviewed scientific journals and presented at clinical and scientific congresses.

Trial registration number NCT04162613.

\section{BACKGROUND}

Anterior cruciate ligament (ACL) injury is a devastating injury with major consequences for the individual including functional limitations, poor quality of life and an increased risk of early-onset knee osteoarthritis. ${ }^{1-5}$ Despite
Strengths and limitations of this study

- There is a lack of studies as the present one that identify modifiable risk factors for secondary anterior cruciate ligament (ACL) injuries.

- The use of not only patient-reported outcomes but also a comprehensive test battery of sensorimotor function to identify risk factors that may be modifiable by training, will shed new light on optimisation of rehabilitation protocols to substantially reduce further injuries after ACL injury.

- The prospective design and the inclusion of participants both in Northern and Southern Sweden will facilitate broad and longitudinal investigation of risk factors for secondary knee injuries after ACL injury.

- The prospective design of this study may constitute a risk of drop-out at follow-up which potentially may have implications for data analysis and, subsequently, interpretation of findings.

- While we will adjust for possible demographic confounders, it will not be possible to adjust for extrinsic confounders, for example, playing surface, which may have implications for the analysis and interpretation of the result.

thorough rehabilitation, it takes approximately 2 years for these patients to regain preinjury function. ${ }^{6}$ Many persons still suffer from persistent functional limitations several years after injury ${ }^{1-4}$ and may never return to their preinjury activity level. ${ }^{6}$ In Sweden, around $50 \%$ of the ACL injuries are treated with surgical reconstruction of the ACL. ${ }^{7}$ However, the risk of rerupture of the ACL after reconstruction is high, and is suggested to be greatest within 2years following ACL reconstruction (ACLR). ${ }^{8}$ The overall reinjury rate is estimated to be around $7 \%$ in Sweden ${ }^{9}$ but in a systematic review on international studies it was reported to be as high as $23 \%$ in younger individuals involved in sport on an elite level. ${ }^{10} \mathrm{~A}$ second ACL injury to either 
knee may have devastating consequences for this group of patients. This may present as a further decline in function $^{11}$ and quality of life ${ }^{1213}$ as well as further increasing the risk of future radiographic knee osteoarthritis ${ }^{14} 15$ compared with individuals with a unilateral injury. Yet, the factors that may predispose some individuals for recurrent knee injuries are not well established.

Return to sport (RTS) too early after ACLR has been reported as a major reason for the observed high reinjury rate. ${ }^{16}$ This may be due to that the individuals may return to their previous activity before gaining adequate functional capacity and physical readiness to RTS, rather than the specific timing of RTS. ${ }^{17-19}$ Several sensorimotor factors, such as poor proprioception, kinematic asymmetry and deteriorated muscular activation pattern, have been theorised to contribute to secondary ACL injury. ${ }^{18}$ Moreover, previous studies have reported reduced lower extremity strength, ${ }^{20}$ altered trunk and knee biomechanics ${ }^{20}$ as well as restricted hip rotation ${ }^{20}$ and ankle range of motion $(\mathrm{ROM})^{21}$ to be linked to sustaining a primary lower extremity injury. Despite this, a recent systematic review (of ours) shows that research on the contributing risk factors for secondary ACL injuries have mainly focused on demographic factors such as age, sex, family history and body mass index, while studies including functional sensorimotor measures as risk factors for reinjury are limited..$^{22}$ Studies on the association between factors that are modifiable by training, and the risk of secondary injuries are thus highly warranted. In this prospective cohort study, we aim to identify risk factors for subsequent ACL injuries that are modifiable by training, in order to optimise training and rehabilitation after ACLR to minimise the risk of reinjury and secure a safer RTS. Specifically, we want to determine if poor sensorimotor function at baseline (1 year post-ACLR) can predict graft rupture within 3 years following ACLR. Secondary aims are to study the association between sensorimotor function at baseline and (i) the risk of contra-lateral ACL injury and (ii) failure to RTS within the same time period.

\section{METHODS AND ANALYSIS}

\section{Study design}

A prospective cohort study, adhering to the Strengthening the Reporting of Observational Studies in Epidemiology guidelines (www.strobe-statement.org).

\section{Participants}

Two hundred participants (50\% women) who have had ACLR will be consecutively recruited from the Clinics of Orthopedics, Norrlands University Hospital and Clinics of Sports Medicine, Umeå, Sweden (n 100) and from an ongoing study (SHIELD (NCT03473873)) including patients recruited from the Department of Orthopedics, Skåne University Hospital, Sweden (n 100). Inclusion criteria: (i) approximately 1 year after surgical reconstruction of the ACL, with or without associated injuries to other structures of the knee, (ii) 15-35 years old. Exclusion criteria: (i) previous ACL injury to either knee; (ii) less than 8 months or more than 16 months after ACLR; (iii) diseases or disorders over-riding the knee condition (eg, neurological disease); (iv) not understanding the language of interests (any Scandinavian language or English). All participants will provide a written informed consent.

\section{Procedure}

The participants will be assessed with a comprehensive test battery for sensorimotor function (hop tests, muscle strength, muscle activation, ROM, postural orientation, proprioception as joint position sense (JPS) test) and patient-reported outcomes at 1-year post surgery (baseline) as described in table 1 (full description of all collected data is given). The same researcher (AC) will be carrying out data collection in Umeå and in Lund and will give standardised verbal instructions. ROM, proprioception and strength tests will be performed without shoes whereas the postural orientation tasks and hop tests will be performed with training shoes. Both the injured and the non-injured legs will be assessed for sensorimotor function for all tests except for muscle activation pattern for which only the injured leg will be assessed. Irrespective of which knee is the injured knee, the right leg will be tested first (except for isokinetic knee strength for which the non-injured leg will be tested first). A warm up of 5 min of stationary cycling, 10 squats, 10 jumps on both legs and 5 jumps on the right and left legs, respectively ${ }^{3}$ precedes the tests.

\section{Predictors}

The Limb Symmetry Index ((LSI) calculated as dividing the result for the injured leg by that of the non-injured leg and multiplying by 100) for the single-leg hop for distance (SLHD), will be the main predictor. Absolute hop distance, ROM measures, muscle strength, muscle activation, proprioception, postural orientation and patient-reported outcomes will be the secondary predictors. Collection of patient-reported outcomes and assessment of sensorimotor muscle function will be carried out in the order described later.

\section{Patient-reported outcomes}

Data for the following valid questionnaires will be collected and managed using an electronic data capture tool (REDCap) prior to the functional testing ${ }^{23}$; for patientreported knee specific function, the Knee injury and Osteoarthritis Outcome Score (all subscales),${ }^{24}$ the Anterior Cruciate Ligament-Quality of Life questionnaire ${ }^{25}$ and Global Knee Function ${ }^{26}$ will be used. For assessment of preinjury and current activity level the Tegner Activity Scale ${ }^{27}$ will be used and for assessing psychological readiness to RTS the ACL RTS after injury ${ }^{28}$ will be used. The Perceived Stress Scale ${ }^{29}$ will be used to evaluate the participants' self-perceived stress.

Hip and ankle ROM

Passive hip ROM will be measured with a digital inclinometer (Commander Echo, JTECH Medical, Salt Lake City, 
Table 1 Demographics, patient-reported outcomes and sensorimotor factors to be collected at baseline (1-year post-ACLR)

\begin{tabular}{|c|c|c|}
\hline Demographics & Patient-reported questionnaires & Sensorimotor function \\
\hline Sex & $\begin{array}{l}\text { Tegner activity scale (preinjury and } \\
\text { current) }\end{array}$ & Hop performance (SLHD (cm, LSI), SH (n, LSI) \\
\hline Age (years) & $\begin{array}{l}\text { Knee injury and Osteoarthritis } \\
\text { Outcome Score (all subscales) }\end{array}$ & $\begin{array}{l}\text { Knee, hip and trunk isometric peak muscle torque } \\
(\mathrm{Nm} / \mathrm{kg})\end{array}$ \\
\hline Height (cm) & ACL quality of life & Knee isokinetic peak torque $(\mathrm{Nm} / \mathrm{kg})$ \\
\hline Body mass $(\mathrm{kg})$ & $\begin{array}{l}\text { Global knee function: numeric } \\
\text { rating scale, } 1 \text { (worst) to } 10 \text { (best) }\end{array}$ & Hip and ankle ROM (degrees) \\
\hline Primary sport & RTS (yes/no, level) & $\begin{array}{l}\text { Postural orientation errors during the SLMS, FL, } \\
\text { SD and SLHD (visual movement quality scoring) }\end{array}$ \\
\hline Years in primary sport & Perceived Stress Scale-10 & $\begin{array}{l}\text { *Kinematics and kinetics during the SLMS, FL, SD } \\
\text { and SLHD (three-dimensional motion analysis) }\end{array}$ \\
\hline Injured knee (left/right) & ACL- RSI & *Proprioception (JPS (degrees)) \\
\hline
\end{tabular}

Graft type

Injury situation (game/practice/other)

Contact/non-contact injury

Associated injuries (eg, collateral

ligament, meniscal injury) to the index

knee (from medical records)

Current pain (numeric rating scale, 0

(none) to 10 (worst))

Family history of $\mathrm{ACL}$ injury

${ }^{*}$ Assessed only in Umeå.

ACL, anterior cruciate ligament; ACLR, ACL reconstruction; FL, forward lunge; JPS, joint position sense; ROM, range of motion; RSI, Return to Sport after Injury; RTS, return to sport; SD, stair descending; SH, sidehop; SLHD, single leg hop for distance; SLMS, single leg mini squat.

Utah, USA). Internal and external hip rotation will be measured with the patient in a seated position with $90^{\circ}$ of flexion in the hip and knee joints. Maximal passive medial and lateral rotation ROM, respectively, will be recorded when the assessor visually observes a lateral pelvic tilt. The mean of two tests will be used in the analysis. ${ }^{30}$ Ankle dorsi-flexion ROM will be assessed in a standing position according to a previously described method. ${ }^{31}$ The ankle dorsiflexion ROM will be assessed with a standard plastic goniometer. The mean of three subsequent measurements will be used for statistical analysis.

\section{Proprioceptive ability}

Participants recruited in Umeå will be assessed for proprioceptive ability using a weight-bearing JPS test developed at Umeå University. ${ }^{32}$ Prior to the assessment, reflective markers will be placed on anatomical landmarks and the knee angle will be calculated with a three-dimensional movement analysis system (Oqus cameras, Qualisys AB, Gothenburg, Sweden). Three practice trials will be performed before testing, and five trials on each leg will then be performed. The mean knee joint angle error resulting from the five trials will be used in the analysis.
Good reliability has been reported for this measurement (intraclass correlation coefficient (ICC) $\left.{ }_{3.1}=0.72\right)^{32}$

Isometric muscle strength

Isometric peak force $(\mathrm{N})$ of the knee, hip and trunk will be measured with a hand held dynamometer (Power Track II Commander Echo, JTECH Medical, Salt Lake City, Utah, USA). The leverage (m) will be measured from the joint axis of rotation to the point of application of the force transducer for each test. The peak value of three trials will then be calculated to Newton metre $(\mathrm{Nm})$, by dividing the peak force value with the corresponding leverage. For analysis, all values will be normalised to body weight $(\mathrm{Nm} / \mathrm{kg})$. Good inter-reliability and intrareliability has been reported for these measurements (ICCs 0.86-0.91, SEM\% 9.5-14.4). ${ }^{33}$

\section{Postural orientation}

Visual assessment

The test battery consists of five functional tasks; singlelimb mini squat (SLMS), stair descending (SD), forward lunge (FL), SLHD and side hop (SH) ${ }^{34}$ The participants will perform the SLMS and SD five times. The FL and SLHD will be performed three times and maximum hops 
in $30 \mathrm{~s}$ for the $\mathrm{SH}$. The tasks will be video-recorded from a frontal view for later assessment of the patient's postural orientation. The videos can be viewed several times and/or in slow motion if needed. Between two and six segment-specific postural orientation errors (POEs) will be observed and scored for each task. The total knee joint POE across all tasks and the total POE score will be used in the analysis. One to two test trials will be allowed for each task. High inter-rater reliability has been reported for this test battery in patients with ACL injury. ${ }^{34} 35$

\section{Three-dimensional motion analysis}

The participants from Umeå will be assessed for threedimensional kinematics with a three-dimensional motion analysis system with eight infrared cameras (Oqus, Qualisys $\mathrm{AB}$ ) during all postural orientation tasks (SLMS, FL, SD, SLHD, SH). Forty-four reflective markers will be attached to anatomical landmarks on the shoulders, torso and lower extremities. One assessor (AC) will perform all landmark palpations and marker attachments to ensure standardisation. Spatial and temporal kinematic and kinetic variables for the knee, hip, pelvis and trunk will be calculated.

\section{Isokinetic muscle strength}

Maximal isokinetic concentric muscle strength tests during knee extension and flexion will be measured at $60^{\circ} / \mathrm{s}$ with a dynamometer (Biodex Medical Systems, Shirley, New York). Five test repetitions will be performed and recorded. Normalised peak torque $(\mathrm{Nm} / \mathrm{kg})$ for knee extension and flexion will be used in the analysis. ${ }^{36}$

\section{Hop tests}

Two commonly used and reliable hop tests, the SLHD and the $\mathrm{SH}$ will be performed as described by Gustavsson et $a l .{ }^{37}$ The SLHD will be performed three times and the distance in centimetres from the toe at the push-off to the heel at the landing position will be measured. The $\mathrm{SH}$ is performed once on each leg and the maximum number of jumps in $30 \mathrm{~s}$ will be recorded. Absolute values for the injured leg and the LSI for both hop tests will be calculated and used in the analysis.

\section{Muscle activation pattern}

Muscle activation timing and preactivation amplitudes will be collected using surface electromyography (EMG) electrodes with a wireless EMG system (Desktop DTS, Noraxon U.S.A., Scottdale, Arizona, USA), with a sampling frequency of $1500 \mathrm{~Hz}$ and a lowpass filter of $500 \mathrm{~Hz}$. Data will be collected for the following muscles during the landing of the SLHD (injured side only); gluteus maximus, gluteus medius, semitendinosus, biceps femoris, vastus medialis, medial gastrocnemius and iliocostalis (bilaterally). All electrodes will be placed according to the SENIAM (surface EMG for non-invasive assessment of muscles) guidelines ${ }^{38}$ and all values will be normalised to each participants maximum voluntary contraction (MVC). MVC for all muscles will be synchronously collected with the peak torque data described earlier and calculated from the maximum value of three repetitions.

\section{Outcomes}

At follow-up at 2 years (3 years post-ACLR), the participants will be contacted by email/phone and asked to answer questions regarding any new ACL injuries and RTS details.

\section{Primary outcome}

Rupture of the reconstructed ACL within 2years from inclusion (self-reported, dichotomous reply (yes/no)).

\section{Secondary outcomes}

1. ACL injury to the contra-lateral knee within 2 years from inclusion (self-reported, dichotomous reply (yes/no)).

2. Failure to return-to-sport after ACLR. The participants will be asked to answer a questionnaire regarding if they plan to return/have returned to the same activity or different activity or activity level as well as time point of return.

\section{Statistical analysis}

Separate logistic regression models, adjusting for possible confounders (eg, sex, age), will be used to evaluate the influence of the different sensorimotor predictors, respectively, on the prospective outcomes (graft rupture, contra-lateral ACL injury and RTS). With the LSI for the single-leg hop test as main predictor and graft rupture as main outcome, power calculations show that with a graft rupture rate of $7 \%$, an estimated OR of 0.95 for the SLHD, power $=80 \%$ and alpha $=5 \%$, at least 174 individuals are needed. Based on this calculation, we will include 200 individuals allowing for an approximate drop-out of $10 \%$.

\section{Patient and public involvement}

This study incorporates previously validated objective and subjective outcomes for this particular group of patients. Patients were not invited to comment on the study design, develop patient-relevant outcomes or interpret the results.

\section{Ethics and dissemination}

This study is approved by the Swedish Ethical Review Board (Dnr 2016/319 and Dnr 2019-04037). The results will be published in international peer-reviewed scientific journals. In addition, the results will be presented in appropriated clinical and scientific congresses.

\section{Time line}

The recruitment of participants started in March 2018 and is expected to continue until March 2022.

Correction notice This article has been corrected since it first published. The provenance and peer review statement has been included.

Twitter Anna Cronström @AnnaCronstrm1

Contributors AC contributed to the conception and design of the study and was in charge of drafting and writing the study protocol. EA and CKH contributed to the 
conception and design of the study and provided feedback on drafts of this paper. All authors read and approved the final manuscript.

Funding This work was supported by the Swedish Research Council for Sports Science (P2019-0011, D2019-0005), The Gyllenstierna Krapperup's Foundation and Lennander's Foundation.

Competing interests None declared.

Patient consent for publication Not required.

Provenance and peer review Not commissioned; externally peer reviewed.

Open access This is an open access article distributed in accordance with the Creative Commons Attribution Non Commercial (CC BY-NC 4.0) license, which permits others to distribute, remix, adapt, build upon this work non-commercially, and license their derivative works on different terms, provided the original work is properly cited, appropriate credit is given, any changes made indicated, and the use is non-commercial. See: http://creativecommons.org/licenses/by-nc/4.0/.

ORCID iD

Anna Cronström http://orcid.org/0000-0002-3282-6320

\section{REFERENCES}

1 Tengman E, Brax Olofsson L, Nilsson KG, et al. Anterior cruciate ligament injury after more than 20 years: I. physical activity level and knee function. Scand J Med Sci Sports 2014;24:e491-500.

2 Whittaker JL, Woodhouse LJ, Nettel-Aguirre A, et al. Outcomes associated with early post-traumatic osteoarthritis and other negative health consequences 3-10 years following knee joint injury in youth sport. Osteoarthritis Cartilage 2015;23:1122-9.

3 Ageberg E, Thomeé R, Neeter C, et al. Muscle strength and functional performance in patients with anterior cruciate ligament injury treated with training and surgical reconstruction or training only: a two to five-year followup. Arthritis Rheum 2008;59:1773-9.

4 Tengman E, Brax Olofsson L, Stensdotter AK, et al. Anterior cruciate ligament injury after more than 20 years. II. concentric and eccentric knee muscle strength. Scand J Med Sci Sports 2014;24:e501-9.

5 Poulsen E, Goncalves GH, Bricca A, et al. Knee osteoarthritis risk is increased 4-6 fold after knee injury - a systematic review and metaanalysis. Br J Sports Med 2019;53:1454-63.

6 Ardern CL, Taylor NF, Feller JA, et al. Fifty-five per cent return to competitive sport following anterior cruciate ligament reconstruction surgery: an updated systematic review and meta-analysis including aspects of physical functioning and contextual factors. $\mathrm{Br} J$ Sports Med 2014;48:1543-52.

7 The Swedish National Knee Ligament Registry. Annual report. Available: https://www.aclregister.nu/

8 Paterno MV, Rauh MJ, Schmitt LC, et al. Incidence of second ACL injuries 2 years after primary ACL reconstruction and return to sport. Am J Sports Med 2014;42:1567-73.

9 The Swedish National Knee Ligament Register. Annual report, 2018.

10 Wiggins AJ, Grandhi RK, Schneider DK, et al. Risk of secondary injury in younger athletes after anterior cruciate ligament reconstruction: a systematic review and meta-analysis. Am J Sports Med 2016;44:1861-76.

11 Fältström A, Hägglund M, Kvist J. Patient-Reported knee function, quality of life, and activity level after bilateral anterior cruciate ligament injuries. Am J Sports Med 2013;41:2805-13.

12 Filbay SR, Ackerman IN, Russell TG, et al. Return to sport matterslonger-term quality of life after ACL reconstruction in people with knee difficulties. Scand J Med Sci Sports 2017;27:514-24.

13 Filbay SR, Culvenor AG, Ackerman IN, et al. Quality of life in anterior cruciate ligament-deficient individuals: a systematic review and meta-analysis. Br J Sports Med 2015;49:1033-41.

14 Pinczewski LA, Lyman J, Salmon LJ, et al. A 10-year comparison of anterior cruciate ligament reconstructions with hamstring tendon and Patellar tendon autograft: a controlled, prospective trial. Am J Sports Med 2007;35:564-74.

15 Wasserstein D, Huston LJ, Nwosu S, et al. KOOS pain as a marker for significant knee pain two and six years after primary ACL reconstruction: a multicenter orthopaedic outcomes network (moon) prospective longitudinal cohort study. Osteoarthritis Cartilage 2015;23:1674-84.
16 Grindem H, Snyder-Mackler L, Moksnes H, et al. Simple decision rules can reduce reinjury risk by $84 \%$ after $A C L$ reconstruction: the Delaware-Oslo ACL cohort study. Br J Sports Med 2016;50:804-8.

17 Fältström A, Hägglund M, Magnusson H, et al. Predictors for additional anterior cruciate ligament reconstruction: data from the Swedish national ACL register. Knee Surg Sports Traumatol Arthrosc 2016;24:885-94.

18 Swärd P, Kostogiannis I, Roos H. Risk factors for a contralateral anterior cruciate ligament injury. Knee Surg Sports Traumatol Arthrosc 2010;18:277-91.

19 Grindem H, Engebretsen L, Axe M. Activity and functional readiness, not age, are the critical factors for second anterior cruciate ligament injury - the Delaware-Oslo ACL cohort study. Br J Sports Med 2020.

20 Hewett TE, Myer GD, Ford KR, et al. Mechanisms, prediction, and prevention of ACL injuries: Cut risk with three sharpened and validated tools. J Orthop Res 2016;34:1843-55.

21 Verrelst R, Van Tiggelen D, De Ridder R, et al. Kinematic chainrelated risk factors in the development of lower extremity injuries in women: a prospective study. Scand J Med Sci Sports 2018;28:696703.

22 Cronström A, Tengman E, Häger CK. Risk factors for contra-lateral secondary anterior cruciate ligament injury: a systematic review with meta-analysis. Accepted to Sports Medicine 2020

23 Harris PA, Taylor R, Thielke R, et al. Research electronic data capture (REDCap)--a metadata-driven methodology and workflow process for providing translational research informatics support. J Biomed Inform 2009;42:377-81.

24 Collins NJ, Prinsen CAC, Christensen R, et al. Knee injury and osteoarthritis outcome score (KOOS): systematic review and meta-analysis of measurement properties. Osteoarthritis Cartilage 2016;24:1317-29.

25 Mohtadi N. Development and validation of the quality of life outcome measure (questionnaire) for chronic anterior cruciate ligament deficiency. Am J Sports Med 1998;26:350-9.

26 Williamson A, Hoggart B. Pain: a review of three commonly used pain rating scales. J Clin Nurs 2005;14:798-804.

27 Tegner Y, Lysholm J. Rating systems in the evaluation of knee ligament injuries. Clin Orthop Relat Res 1985;198:42-9.

28 Kvist J, Österberg A, Gauffin H, et al. Translation and measurement properties of the Swedish version of ACL-Return to sports after injury questionnaire. Scand J Med Sci Sports 2013;23:568-75

29 Taylor JM. Psychometric analysis of the Ten-Item perceived stress scale. Psychol Assess 2015;27:90-101.

30 Pua Y-H, Wrigley TV, Wrigley TW, et al. Intrarater test-retest reliability of hip range of motion and hip muscle strength measurements in persons with hip osteoarthritis. Arch Phys Med Rehabil 2008;89:1146-54.

31 Powden CJ, Hoch JM, Hoch MC. Reliability and minimal detectable change of the weight-bearing lunge test: a systematic review. Man Ther 2015;20:524-32.

32 Lundqvist P. Measuring knee joint position sense: test-retest reliability of a new developed method in a 3D-motion analysis laboratory. Umeå University, 2016.

33 Kemp JL, Schache AG, Makdissi M, et al. Greater understanding of normal hip physical function may guide clinicians in providing targeted rehabilitation programmes. J Sci Med Sport 2013;16:292-6.

$34 \mathrm{Nae}$ J, Creaby MW, Ageberg E. Extended version of a test battery for visual assessment of postural orientation errors: face validity, internal consistency, and reliability. Phys Ther 2020;100:1542-56.

35 Nae J, Creaby MW, Nilsson G, et al. Measurement properties of a test battery to assess postural orientation during functional tasks in patients undergoing anterior cruciate ligament injury rehabilitation. $J$ Orthop Sports Phys Ther 2017;47:863-73.

36 de Araujo Ribeiro Alvares JB, Rodrigues R, de Azevedo Franke R, et al. Inter-machine reliability of the Biodex and Cybex isokinetic dynamometers for knee flexor/extensor isometric, concentric and eccentric tests. Phys Ther Sport 2015;16:59-65.

37 Gustavsson A, Neeter C, Thomeé P, et al. A test battery for evaluating hop performance in patients with an ACL injury and patients who have undergone ACL reconstruction. Knee Surg Sports Traumatol Arthrosc 2006;14:778-88.

38 Hermens HJ, Freriks B, Disselhorst-Klug C, et al. Development of recommendations for SEMG sensors and sensor placement procedures. J Electromyogr Kinesiol 2000;10:361-74. 